\title{
Winterreise op. 89 di Schubert: una lettura a tre chiavi
}

\author{
di Tiziana Canfori \\ tiziana.canfori@conspaganini.it
}

\begin{abstract}
This paper explains the "birth" and development of an idea of performance: "Winterreise" by Franz Schubert; the concert was premiered at Levanto Music Festival (La Spezia-Italy) in summer 2021. The performers were Mirella Di Vita (soprano), Tiziana Canfori (pianist) and Simone Fareri (painter and sculptor). The artists conceived a detailed performance that combined music, lyrics and fine arts, intending to get the audience more involved in listening to this long and complex Lieder cycle. The different languages and the space-time arrangement had to be considered in the structure of this concert to create a more natural and complete sharing experience for the audience.
\end{abstract}

Keywords: Musical Journey, Concerted with Fine Arts, Counterpoints

Nel cartellone 2021 del Levanto Music Festival Amfiteatrof è stato inserito uno spettacolo al quale ho partecipato con grande piacere come pianista, ma soprattutto $\mathrm{mi}$ ha dato l'occasione per una profonda e appassionante riflessione sul modo di presentare al pubblico un repertorio ancora difficile da maneggiare per l'ambiente italiano. In programma la Winterreise op. 89 di Franz Schubert, su testi di Wilhelm Müller, su cui abbiamo costruito un'interpretazione che abbiamo chiamato "a tre chiavi" perché inserisce oltre alla voce e al pianoforte anche una lettura in chiave figurativa. Miei compagni di strada in questo esperimento sono stati il soprano Mirella Di Vita e l'artista Simone Fareri.

Vorrei raccontare il nostro percorso e l'esperienza di questo concerto, partendo da alcune premesse necessarie.

Presentare un ampio ciclo liederistico al pubblico italiano è ancora oggi piuttosto difficile, soprattutto in un festival estivo che giustamente mira alla 
piacevolezza più che alla proposta di un faticoso sforzo intellettuale. La nostra avventura era particolarmente difficile: un viaggio d'inverno in pieno agosto, 80 minuti di testi tedeschi calati nelle atmosfere sempre più rarefatte e coraggiose dell'ultimo Schubert, la morte incombente... mentre fuori un sole rovente tramontava sulla spiaggia ancora affollata di ombrelloni aperti e le gelaterie brulicavano di avventori con coni colorati, freschi e colanti. Incontrare quel pubblico era il nostro scopo.

Abbiamo per prima cosa valutato l'impatto dell'elemento vocale. Il pubblico di oggi è abituato a una molteplicità di esperienze d'ascolto della voce e quando pensa alla parola "cantante" può associarla alla musica antica come al teatro musicale, al jazz, al pop, alla canzone d'autore o al Festival di Sanremo. In genere queste esperienze convivono numerose nelle abitudini dell'ascoltatore, che le coltiva secondo i propri gusti.

Resta il fatto che l'uso della voce, in qualsiasi genere musicale, scuote l'ascoltatore con un'energia particolare: la presenza di uno strumento così fisico e immediato suscita la partecipazione spontanea di chi ascolta, portandolo più facilmente a condividere un'emozione. Allo stesso modo il carisma dell'interprete avvolge il pubblico con forza, chiamando in gioco tutti i piani di trasmissione attraverso le caratteristiche fisiche, intellettuali ed emotive di quel corpo cantante. Noi musicisti sappiamo bene che può esistere una profonda capacità di fascinazione immediata anche nella performance di uno strumentista, ma sappiamo anche cosa succede quando nel nostro gruppo inseriamo un cantante: il pubblico si attiva e reagisce maggiormente, dalle risa incontenibili dei bambini quando vengono a visitare il Dipartimento di Canto in Conservatorio, alle lacrime che scopriamo negli occhi di chi assiste alla morte di Mimì o di Violetta. Emozioni forti, insomma. Ed è certo che al mondo è più conosciuto il fazzolettone sventolato dalle mani di Pavarotti che non le minigonne vertiginose di Yuja Wang.

Il canto come scelta vincente? Non del tutto e non sempre, come sa chi si occupa di Liederistica alle nostre latitudini. Sembra che in questo caso il 
destino si inverta. $\grave{E}$ tuttora difficile far penetrare questo repertorio in una cultura che alla voce chiede altre emozioni. Sembra che il fatto stesso di avere una linea significante in più, cioè un testo poetico che chiede di essere ascoltato, renda l'approccio più difficile e più "mentale". Un repertorio da intenditori, insomma, idealmente contrapposto a quello della tradizione italiana più popolare, che vede la voce come espressione diretta e spontanea. Basta frequentare una classe di canto per comprendere quanto questa spontaneità sia in realtà tutta da conquistare, ma resta forte nell'ascoltatore l'idea che la voce sia un dono di Dio che sgorga direttamente dal cuore. Una grande tradizione d'opera è per il pubblico italiano un tesoro da salvaguardare, ma anche il rischio di un'abitudine pericolosa: vedere l'opera come un luogo comune, oppure a volte come materia da collezionisti (il melomane gira il mondo per seguire le sue stelle e ricordarne tutte le apparizioni) o come gara in cui tifare.

C'è qualcosa che imparenta il teatro d'opera alla gara sportiva: lo sforzo fisico, il gioco di squadra, i grandi spazi, le tifoserie, la pressione della massa degli spettatori, il gesto atletico degli acuti come rigori in campionato... Sto semplificando troppo, lo so, ma la programmazione dei teatri d'opera in tutto il mondo tende a darmi ragione.

Che dire invece quando la voce intona una melodia da camera? La tradizione italiana è ricca anche di canzoni, serenate, barcarole, stornelli: sono forme minori, intime, spesso associate a un ambiente dialettale. Canzoni che si cantano con passione, come quelle dello splendido repertorio napoletano, che certo vanta nobili radici nell'opera settecentesca. Anche questo è un uso della voce che amiamo.

Resta la questione della lingua: una canzone si deve condividere intimamente, va a toccare il cuore, diverte, rattrista, racconta. Il rapporto col testo è fondamentale, anche se tutti abbiamo apprezzato in gioventù anche canzoni in inglese di cui non conoscevamo il testo, scatenati da una musica, in genere ballabile, di cui decretavamo il successo senza analizzare situazioni 
e parole. Anche in questo caso il carisma dell'interprete faceva la parte del leone: insieme alla canzone passava il ciuffo di Elvis, la canottiera di Freddie Mercury, la fisicità trasgressiva di Mick Jagger o quella più morbida, stravagante e colorata di Elton John.

Con buona pace dell'illusione che la musica sia linguaggio universale, ecco che se mettiamo un cantante fermo vicino a un pianoforte, con la bocca piena di parole tedesche, il pubblico tenderà ad allontanarsi e ad annoiarsi, malgrado la presenza della voce.

Se l'opera può assomigliare a una gara sportiva, l'ascolto di un ciclo di Lieder è piuttosto una gara con se stessi, alla ricerca di associazioni, ricordi, equilibri di colori e gusti. Allo stesso modo, la ricerca degli esecutori è volta alla valorizzazione della parola e del suono in ogni sfumatura: l'aspetto attoriale diventa capacità di rendere situazioni e personaggi scavando in profondità nel testo e nella musica, intrecciando col pianoforte un legame fatto di vibrazioni sottili. Si cammina insieme, musicisti e pubblico, come buoni compagni di strada, disegnando un paesaggio sonoro ed emotivo: di questo sono testimonianza i continui riferimenti al "wandern" e al "reisen" in tutto il repertorio tedesco, soprattutto romantico.

Per raggiungere il pubblico c'è quindi da colmare una distanza, che permetta questa gioia di camminare insieme. Come farlo con una lingua ricca e complessa come il tedesco, spesso ingiustamente temuta o rifiutata dal pubblico italiano? In molte stagioni di concerti si sceglie la via delle traduzioni scritte, invitando gli spettatori a seguire il testo leggendo dal programma. Questa soluzione non ci piaceva, perché obbliga il pubblico a staccare gli occhi dalla scena - così importante anche per questo repertorio - per puntarli sul programma: l'attenzione si sposta continuamente, a volte la lettura è difficile per banali questioni di luce o di stampa, il filo si perde... e resta la brutta impressione di essere stati a scuola, più che immersi nella felicità di un concerto. La nostra ricerca era quella di suggestionare, più che di informare, o meglio di informare attraverso la suggestione. 
Abbiamo quindi scelto la via di aggiungere una chiave di lettura che potesse interpretare i dati necessari per seguire il ciclo e insieme aprire una porta a sensazioni personali. Abbiamo trovato in questo la preziosa collaborazione di Simone Fareri, architetto e artista, che interpreta la musica in modo profondo e personale, pur non essendo musicista. Fin dalla prima lettura assieme abbiamo visto che la sua immaginazione cercava nel campo giusto, con libertà creativa, abbandonando completamente il compito dell'illustratore per entrare con noi interpreti nel gioco dell'interpretazione. Per esempio nell'immagine del "Lindenbaum" (Figura 1) non c'era il tiglio che tutti ci aspettavamo, ma un omino perso in una distesa di ghiaccio. In effetti il tiglio del Lied invita a ritornare verso casa, offre un abbraccio che sa di conforto e di morte ("du fändest Ruhe dort"), ma il poeta non cerca di tornare, ormai è immerso nel viaggio e nel proprio destino.

Invece in "Rückblick" (Figura 2) l'immagine si sposa subito alla veemenza affannosa della musica con un fondo nero dal quale un occhio rivive il dolore della partenza uscendo quasi dai frammenti di un'esplosione.

I corpi sono spesso stilizzati, come in "Irrlicht" (Figura 3) dove il personaggio è illuminato e persino contenuto dalla luce che lo guida, oppure pesanti come in "Rast" e "Im Dorfe", perché la fatica e il sonno pesano con dolore e solitudine.

A volte un tratto appena accennato racconta più di molte parole, come accade con la foglia morta di "Letzte Hoffnung" (Figura 4), che è anche diventata il frontespizio dello spettacolo. 


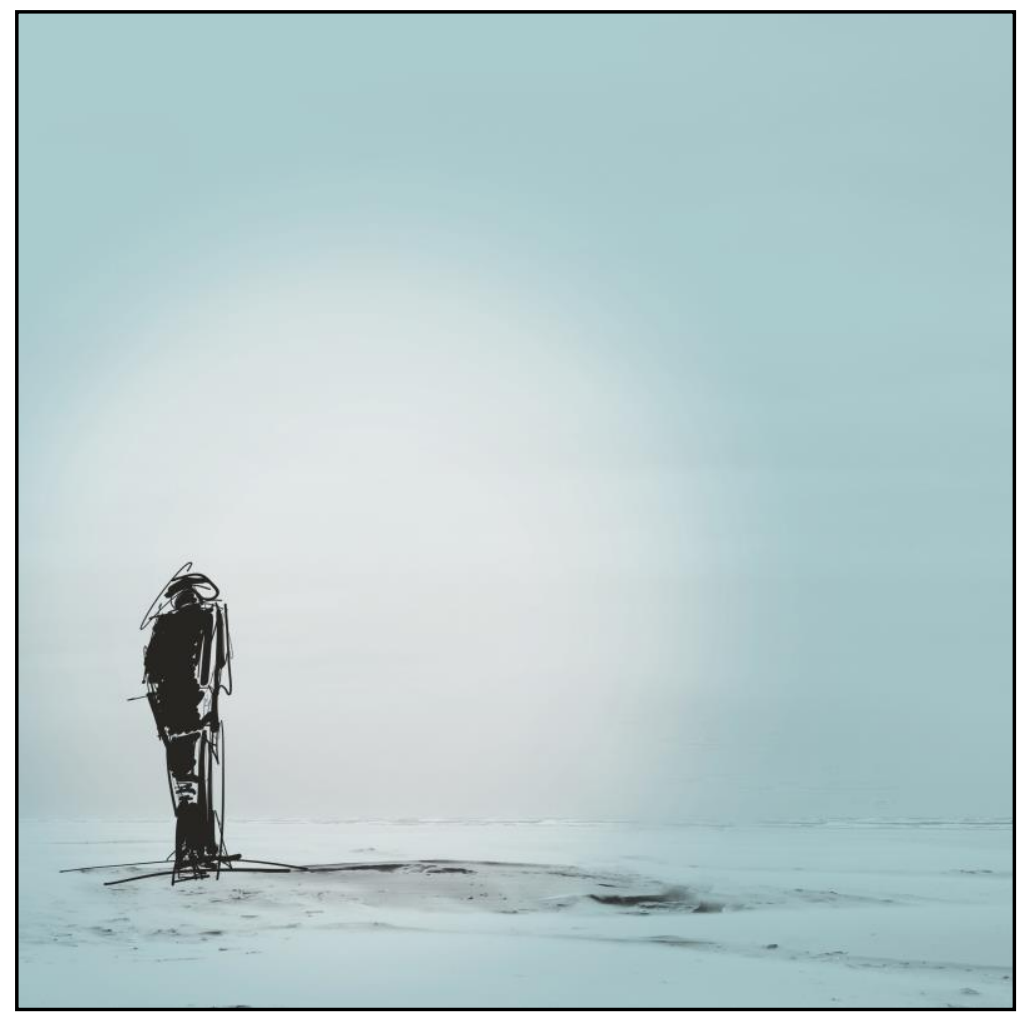

Figura 1: Simone Fareri, Der Lindenbaum.

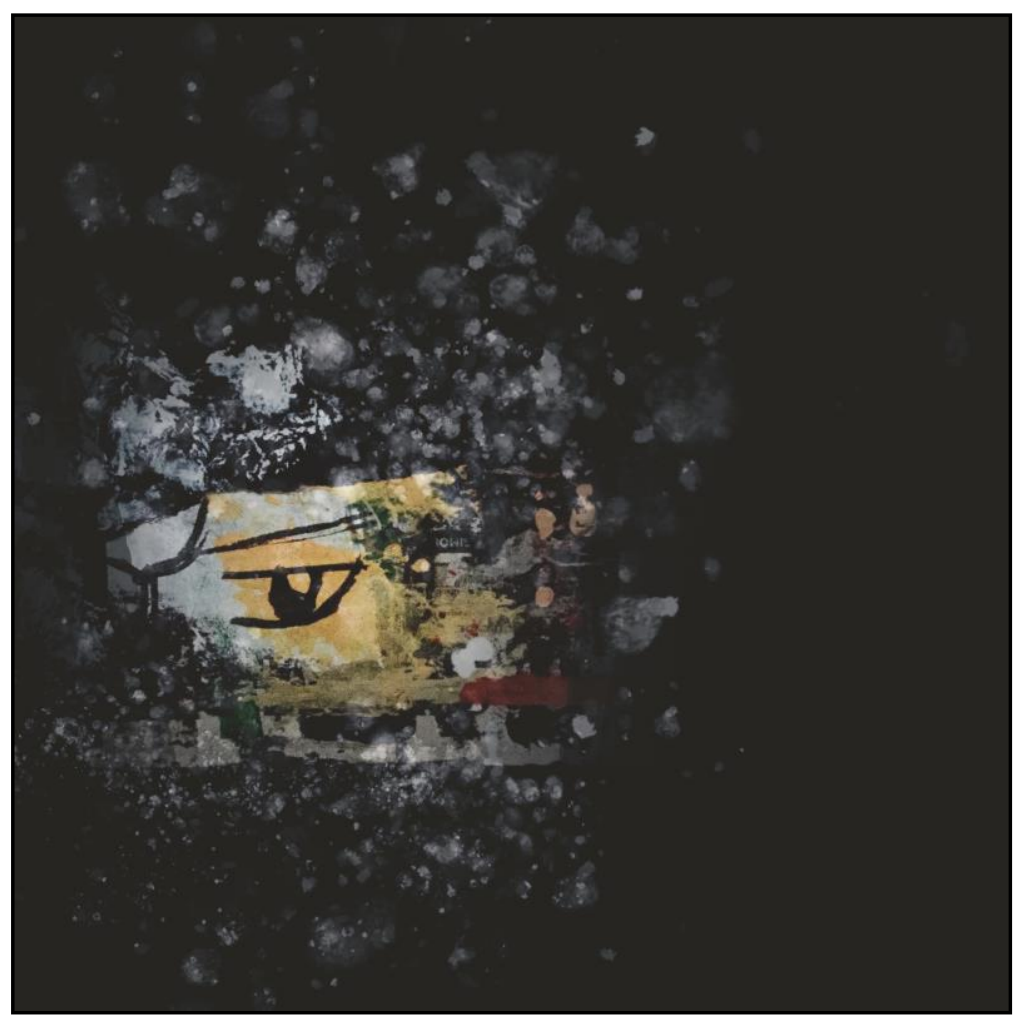

Figura 2: Simone Fareri, Rückblick.

Materiali di Estetica - N. 8.2: 2021, Pagina 450 


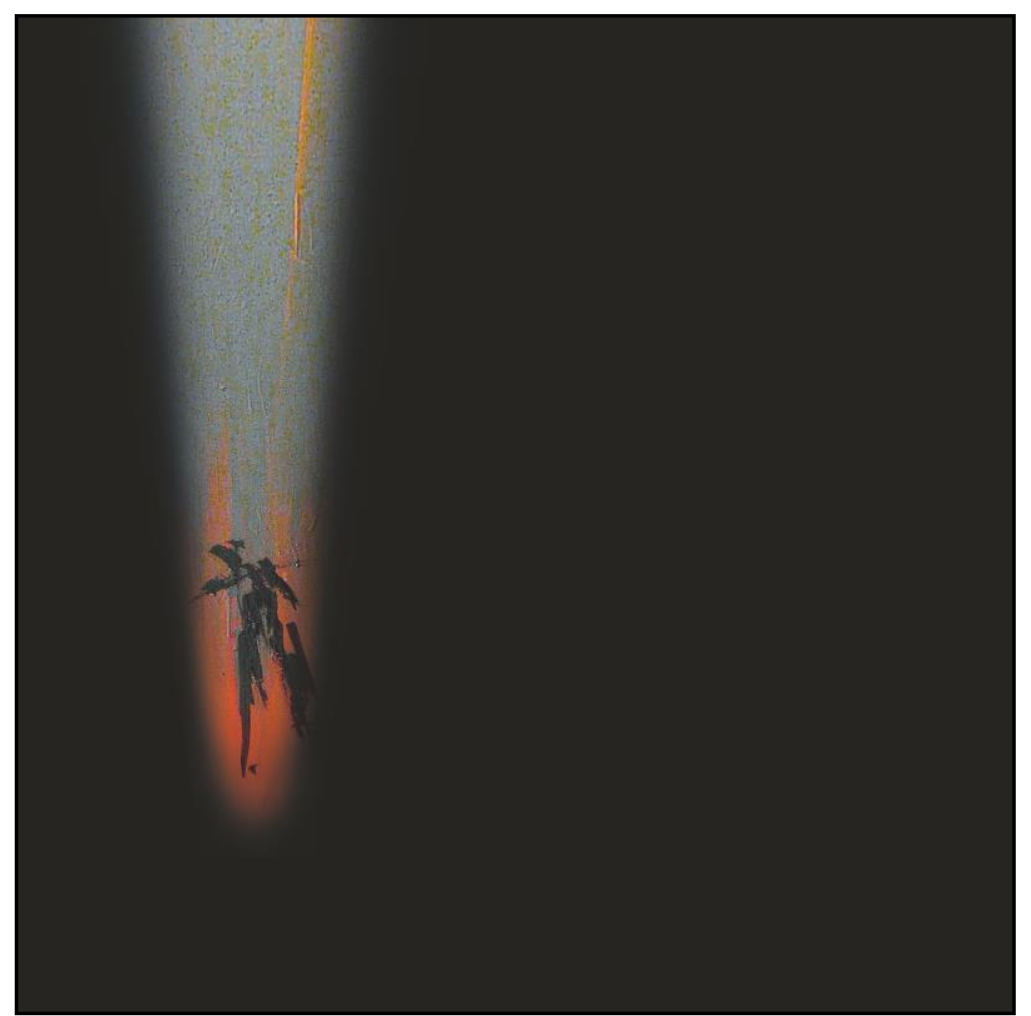

Figura 3: Simone Fareri, Irrlicht.

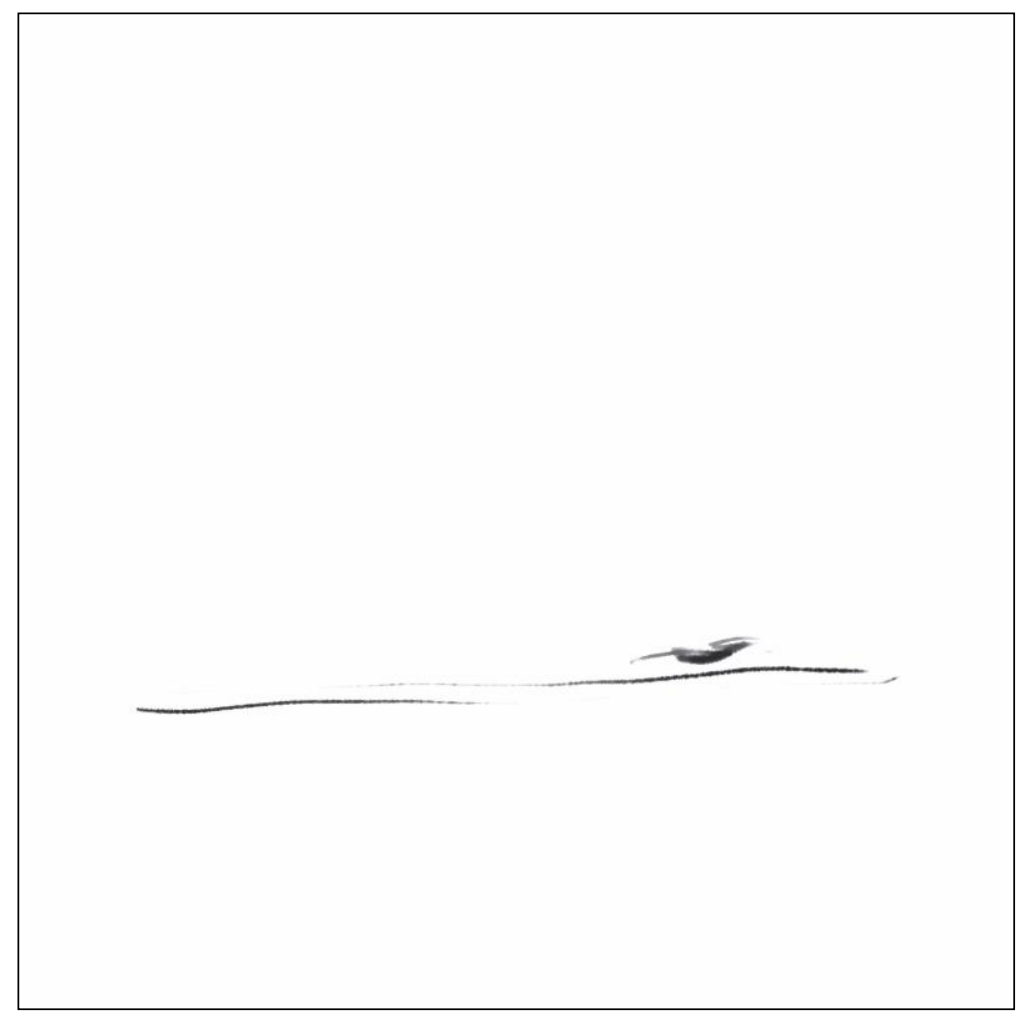

Figura 4: Simone Fareri, Letzte Hoffnung.

Materiali di Estetica - N. 8.2: 2021, Pagina 451 
Ci è apparsa subito concretamente la lettura "a tre chiavi" che andavamo cercando, che poteva giocare sia nella triade testo/musica/immagine, sia in quella che vive fisicamente lo spazio scenico, cantante/pianoforte/schermo.

Questo ci ha portati a modificare una serie di elementi che hanno assunto grande importanza nei nuovi equilibri: la posizione degli interpreti e dello schermo, la luce, la distanza dal pubblico. Una profonda riflessione è stata necessaria intorno alle questioni del tempo, e forse proprio questo è stato il terreno che ci ha dato maggior lavoro e maggiori soddisfazioni. La musica è tessuta nel tempo, come è noto a tutti, ma anche la fruizione del testo e soprattutto dell'immagine si intreccia nell'esperienza d'ascolto a cui abbiamo lavorato. Un ciclo di 24 Lieder era naturalmente una sfida temibile da affrontare: il rischio che la percezione del tempo diventi noia nell'ascoltatore è tangibile. Abbiamo deciso di interpretare tutta l'impresa partendo dalle esigenze della scena e abbiamo inserito in questa guida i tempi delle immagini e delle parole che interagivano con la musica. Un contrappunto, insomma, dove tutte le voci sono ugualmente al servizio dell'insieme, ma dialogano alla pari.

Insieme alle immagini abbiamo inserito prima di ogni brano una slide ben leggibile con una traccia del testo, che permettesse al pubblico di seguire le orme del poeta lungo il suo "viaggio d'inverno" e desse qualche idea degli incontri fatti lungo la strada, dalla casa della sua bella ai vari cani, corvi, cornacchie, ombre di luna, piante, stelle... fino all'apparizione del Leiermann. È sorta per me la necessità di ripensare la traduzione dei testi e di operare una sintesi che rimanesse in linea e sostenesse la curiosità del pubblico fino all'ultimo. Prima di ogni Lied, qualche secondo di silenzio permetteva al pubblico di leggere la situazione raccontata nel brano, senza pretendere di imporre tutto il testo, ma caso mai stimolando una lettura approfondita in un secondo approccio; in quel momento cantante e pianista erano ferme, ma alla sparizione del testo prendeva subito corpo la musica di Schubert e appariva l'immagine che la interpretava, come una sorpresa. 
Molto particolare è stata la nuova dimensione in cui il soprano Mirella Di Vita ed io abbiamo vissuto il rapporto voce-pianoforte. Tradizionalmente pensato per voci più scure, generalmente maschili, il ciclo ha trovato in Mirella un'interprete potente e del tutto credibile (altro miracolo della liederistica, che mette in scena il testo, più che la persona, e permette una libertà di genere molto ricca di possibili intuizioni). Anche gli aspetti tecnici, come la fatica vocale, che certamente preoccupa in un'impresa del genere, sono stati affrontati da lei con uno slancio e una sapienza che traevano vantaggio proprio dalla consapevolezza della maggiore complessità in cui la voce andava a inserirsi. Dal pianoforte, la linea vocale appariva sempre motivata, sicura, coinvolgente. Tutto è sembrato più "giusto", più "facile" e più "condivisibile".

L'esigenza di sintesi ha richiesto un'immersione nuova nei testi, la scelta di elementi portanti e l'uso di un linguaggio lineare e pulito: ogni Lied doveva stare in una slide e i caratteri dovevano essere abbastanza grandi da essere letti a distanza. Ho amato molto questo esercizio, che comunque mi ha permesso di trasmettere meglio la mia interpretazione in alcuni punti che a volte mi appaiono fuorvianti nelle traduzioni correnti. Faccio due esempi, entrambi legati agli ultimi Lieder del ciclo, in cui la parola si fa sempre più sottile e pregnante. 


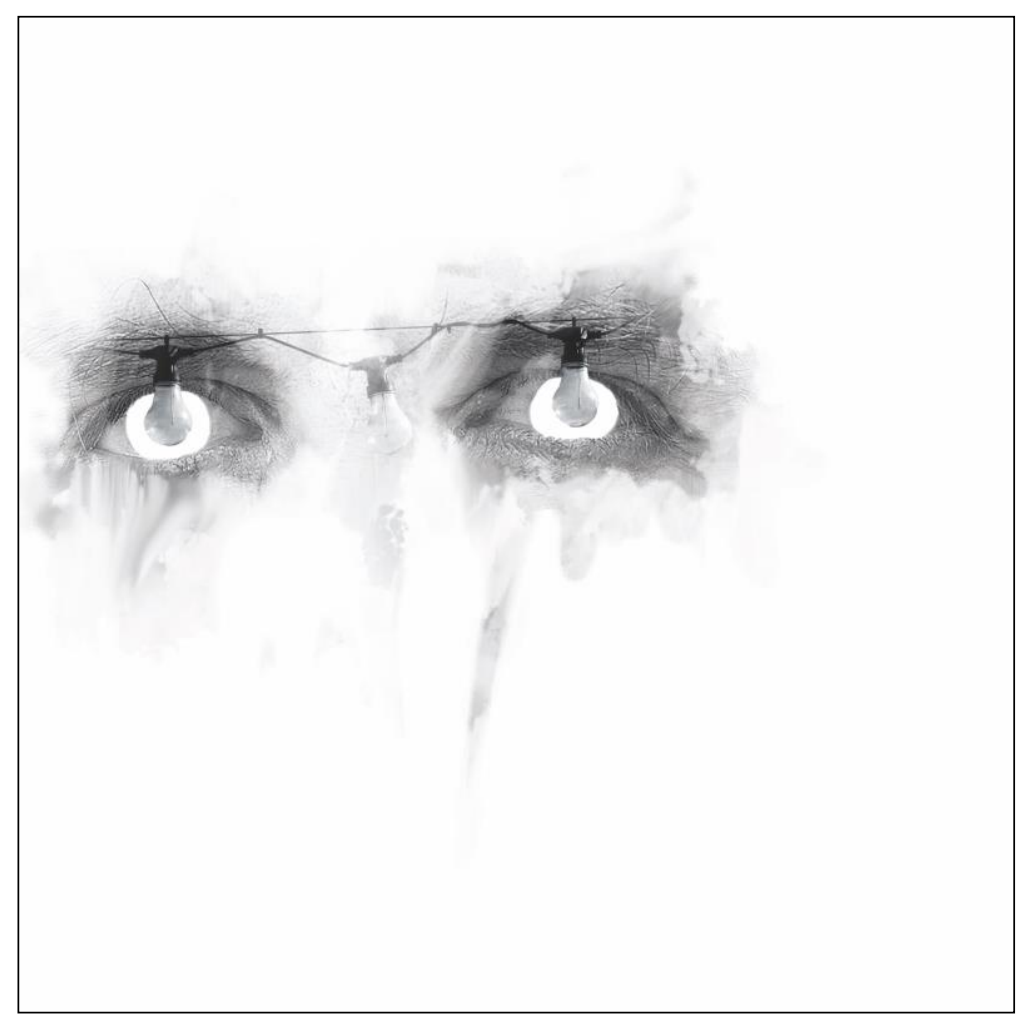

Figura 5: Simone Fareri, Die Nebensonnen.

Nel n. 23 Müller e Schubert ci raccontano di tre soli, "Die Nebensonnen": appaiono fissi e distanti, ma due sono già tramontati e il poeta si augura che sparisca al più presto anche il terzo. "Im Dunkel wird mir wohler sein" dice, conscio con fierezza della propria solitudine e di interpretare l'idea "Will kein Gott auf Erden sein, sind wir selber Götter!" che chiude il Lied precedente, "Mut". Se traduciamo solo il titolo, alla lettera, interpretando "Sonnen" con "soli", rischiamo di andare fuori strada, sia rispetto al testo, sia rispetto alla chiusura del ciclo, che di luce e calore non ha più bisogno. Evidentemente i soli sono astri, punti di riferimento in una volta celeste che ruota fredda e distante, cambia disegno e destino, e che pure rimane compagna di viaggio fino alla fine. Lo spegnersi di quegli astri è lo spegnersi del colloquio con il cielo infinito, è la notte della morte, il coraggio dell'ultimo attimo di solitudine estrema. La parola "sole" non può avere questo sapore, soprattutto alle nostre latitudini, dove il sole è immagine di potenza e unicità. La musica infatti 
interpreta una distanza e una malinconia siderali. Normalmente uso molte parole per introdurre questo Lied ai miei studenti e mi sembrava difficile trasmettere le stesse coordinate al pubblico del concerto. Devo dire che in questo senso la sintesi del testo e soprattutto l'interpretazione artistica di Fareri ci hanno dato un grande aiuto nel suggerire la tensione di questo Lied difficile, che prelude all'ultimo passo.

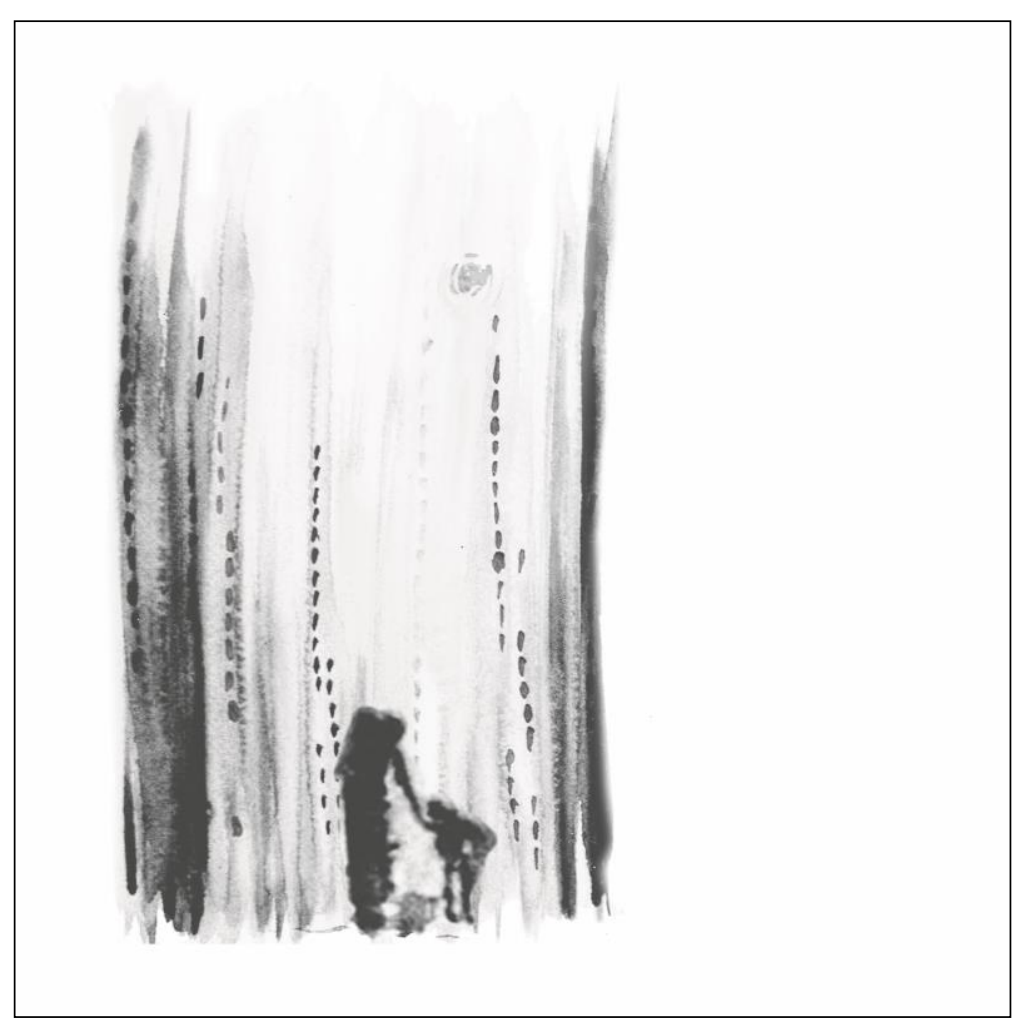

Figura 6: Simone Fareri, Die Leiermann.

L'altro punto in cui ho voluto indicare una scelta è proprio nell'ultimo Lied, dove avviene l'incontro col Leiermann. Perché tradurre "Leier" con "organetto", come spesso leggo? Lo strumento suonato da questo vecchio scalzo è una ghironda, non un organetto. L'errore di traduzione dimostra una scarsa attenzione alla scrittura musicale, che descrive proprio il bordone con la quinta vuota di questo antico strumento popolare a corde strofinate, che nulla ha dell'organetto; anzi, della ghironda viene imitato anche, con l'acciaccatura al basso, lo scatto d'avvio del meccanismo di legno. L'intreccio 
di nomi e significati è comunque interessante: in tedesco si passa attraverso Leier e Drehorgel (organo da girare) per il fatto che la ghironda accompagnava fin dal Medio Evo la musica sacra e soprattutto il canto dei pellegrini, essendo strumento polifonico e insieme molto facile da trasportare. Dal meccanismo a manovella della ghironda si è passati alla manovella dell'organetto da strada, è vero, ma in altre epoche e in altri repertori. Nell'immaginario italiano c'è l'organetto (il Leierkasten) ottocentesco, quello che potremmo immaginare nel secondo quadro di Bohème, per esempio, portatore di un'allegria malinconica agli incroci delle strade cittadine, ma vorrei evitare il rischio di imparentare il Leiermann con la fila di poveri, orfane, reduci, infermi, venditrici di fiori e danzatrici di strada che popolano la musica italiana di fine Ottocento.

Se poi vogliamo perderci, "organetto" è anche uno splendido strumento a mantice presente anche nella tradizione popolare italiana, ma non si traduce con Leier e soprattutto non suona come nel brano di Schubert. La cosa divertente è che questo tipo di organetto nasce proprio a Vienna nel 1829, pochi mesi dopo la morte di Schubert ma, benché dotato di una meccanica piuttosto complessa... di certo non ha mai posseduto una manovella. Perché regalare a quel povero vecchio dignitoso, misterioso e astratto dell'ultimo Lied uno strumento che si sarebbe diffuso qualche decennio dopo, oppure uno strumento su cui il verbo "girare" non ha nessun senso? Vale la pena di ascoltare il suono affascinante di una ghironda per capire che solo quel suono dà il senso di distanza, di uscita dalla vita e dal tempo che Schubert voleva imitare nel capolavoro con cui chiude la Winterreise. E nella slide era meglio lasciare una parola un po' misteriosa piuttosto che suggerire un'immagine sbagliata.

Nella nostra sintesi, quindi, ogni titolo e ogni parola avevano dietro precisi criteri di scelta e una lunga maturazione. In realtà inglobare un tempo di lettura in un'esecuzione musicale non è semplice: noi musicisti siamo abituati a costruire anche i nostri silenzi in funzione esclusiva del tessuto musicale e 
quindi il dover attendere porta rischi per la concentrazione. Abbiamo affrontato questo aspetto con facilità, appena il ritmo dello spettacolo ci è apparso convincente; va detto inoltre che avevamo scelto una figura dietro le quinte, Lorenza Cevasco, cantante e pianista, che era perfettamente in grado di governare al computer sia i tempi per la lettura dei testi, sia le esigenze dell'esecuzione in scena e le immagini.

Nella fase di prova, Mirella Di Vita ed io ci siamo accorte che questa costruzione ci piaceva e ci metteva in una prospettiva di condivisione con il pubblico, così ho potuto mettere a punto, sempre con l'aiuto di Fareri, un altro passaggio a cui tenevo: l'apparizione della "parole sparse". Sull'immagine principale di ogni Lied, che restava ferma per tutto il tempo dell'esecuzione musicale, abbiamo fatto apparire e scomparire delle parole, come in filigrana, che uscivano dal testo ed erano presentate graficamente sia in tedesco, sia nella traduzione italiana. Anche qui l'impegno è stato nella scelta, a volte dettata dall'importanza concettuale delle parole, a volte dalla prepotenza con cui emergevano dalla musica, a volte per la loro suggestiva forma tedesca. Poteva essere che il pubblico riconoscesse quella parola nel corso del Lied, o che ne fosse incuriosito, ma comunque anche questo faceva parte della "terza chiave", che mirava a portare l'ascoltatore, in comodità e in libertà, a gestire meglio la sua esperienza schubertiana.

Il giorno del concerto, questa macchina ci dava fiducia e allegria. Soprattutto ci piaceva di aver aumentato la comodità di ascolto dando il supporto necessario alla fruizione del programma, senza per questo creare una gabbia: gli elementi dello spettacolo erano tutti al loro posto ed erano gestiti in tempo reale, permettendo al pubblico una certa varietà di scelte sulle suggestioni da seguire. Abbiamo così maturato l'ultima idea, figlia delle numerose esperienze sperimentate nei rapporti a distanza imposti dal periodo covid: pochi minuti prima del concerto abbiamo messo sul sito del festival tutte le immagini e tutti i testi, permettendo così al pubblico una soluzione "smart" per portarsi via qualcosa della serata e soprattutto per percorrere il 
concerto liberamente, senza l'ansia di perdere qualcosa di quanto avveniva sullo schermo, ritrovando testi e immagini.

La risposta del pubblico è stata molto incoraggiante. L'attenzione e la comodità d'ascolto si sentivano nella vibrazione positiva che ci arrivava dalla sala, l'esecuzione è stata per noi impegnativa, ma sempre fluida e motivata; il pubblico ci ha ringraziati e soprattutto ci ha dimostrato curiosità per il repertorio, il che vuol dire che non si è sentito estraneo al concerto. La bella voce di Mirella Di Vita è stata apprezzata in modo incondizionato. Gli spettatori, soprattutto quelli delle ultime file, hanno saputo usare i loro telefonini per stare con noi e seguirci meglio, invece che per distrarsi...

L'integrale di Winterreise è sempre un impegno serio, in questo caso ancora di più per la complessità della struttura, ma desideriamo riproporlo. Certo, si tratta di un concerto per cui ci vuole una scena attrezzata e un coordinamento forte, ma portare le straordinarie sfumature schubertiane a conoscenza di un pubblico più vasto è sfida da non abbandonare.

\section{Consigli bibliografici e di ascolto}

GOLDSCHMIDT, Harry, Schubert, Edizioni Ricordi LIM, Milano, 1995.

MOORE, Gerald, I cicli di Lieder di Schubert. Con pensieri sullinterpretazione, Analogon, Asti, 2013.

BOSTRIDGE, Ian, Il viaggio d'inverno di Schubert. Anatomia di un'ossessione, Il Saggiatore, Milano, 2015.

Rimane sempre valido il riferimento a due grandi interpreti del passato:

- Dietrich Fischer-Dieskau, di cui segnalerei le edizioni con Gerald Moore (Deutsche Grammophon), Alfred Brendel (Philips) e Daniel Barenboim (Deutsche Grammophon);

- Christa Ludwig, nella registrazione con James Levine (Deutsche Grammophon). 
Molto interessanti sono anche quattro interpretazioni più recenti:

- Thomas Quasthoff e Charlse Spencer (RCA);

- Jonas Kaufmann e Helmut Deutsch (Sony).

Segnalo infine i link di due esecuzioni a cui sono particolarmente affezionata, avendole potute ascoltare anche dal vivo:

- Matthias Goerne con Christoph Eschenbach (https://youtu.be/mO5RA VJGCJk);

- Ian Bostridge con Julius Drake (https://youtu.be/iRXFyDro4xQ).

Non mi risultano CD di Winterreise con Goerne, mentre Bostridge ha registrato con Drake anche un interessante DVD: Winterreise, con regia di David Alden, per NVC Arts - Warner Music Vision. 